\title{
Metabolic and Endocrine Profiles in Sick Neonatal Foals Are Related to Survival
}

\author{
L. Armengou, E. Jose-Cunilleras, J. Ríos, C. Cesarini, J. Viu, and L. Monreal
}

\begin{abstract}
Background: Sick neonatal foals suffer from a variety of endocrine and metabolic derangements that may be related to outcome. There are several hepatic and lipid metabolism blood markers that have never been assessed in neonatal foals.

Objectives: Assess panel of endocrine and metabolic variables in group of sick and healthy neonatal foals in order to describe their relationship with diagnosis and survival.

Animals: All neonatal foals referred to Unitat Equina-Fundació Hospital Clínic Veterinari during 3 consecutive foaling seasons and a group of healthy foals.

Methods: Observational prospective study. Blood samples were obtained on admission and, when possible, after 24-48 h of hospitalization and immediately before discharge or death. Measured variables were triglycerides, nonsterified fatty acids, glucose, creatinine, urea, $\gamma$-glutamyltransferase, glutamate dehydrogenase (GLDH), insulin, cortisol, bile acids, and adrenocorticotropic hormone (ACTH). ACTH/cortisol and glucose/insulin ratios were calculated.

Results: Urea, creatinine, and cortisol had median concentrations in septic and nonseptic foals 2- to 8-fold higher than in the control group $(P<.001)$. Median ACTH concentration in the septic group was approximately 4 times higher than in nonseptic and control foals $(P<.001)$. ACTH/cortisol ratio was significantly lower in sick foals compared to control foals $(P<.001)$. A score was designed including creatinine, GLDH, and cortisol. When $\geq 2$ of these variables were altered $(P<.001)$, the foal had 32 times more risk of dying (OR, 31.7; 95\% CI, 7.7-130.3).

Conclusions and Clinical Importance: Plasma creatinine, GLDH, and cortisol should be determined in sick newborn foals on admission because of their association with survival.
\end{abstract}

Key words: CIRCI; hypertriglyceridemia; neonatal septicemia; prognosis.

$\mathbf{I}^{\mathrm{n}}$ $\mathrm{n}$ neonatal foals, inflammation and infection are associated with several metabolic and endocrine derangements. ${ }^{1-6}$ These disorders are related to what is called systemic inflammatory response syndrome (SIRS) and are considered an adaptative response. When the inflammatory response is prolonged, however, it triggers a sustained and irreversible catabolic state mediated by release of cytokines and by activation of the hypothalamic-pituitary-adrenal (HPA) axis. ${ }^{1,6}$

In 2008, a consensus statement in human critical care medicine recommended the use of the term critical illness-related corticosteroid insufficiency (CIRCI), instead of relative adrenal insufficiency (RAI), referring to the dysfunction of the HPA axis in the context of critical illness. ${ }^{7}$ In recent years, it has been determined that illness such as trauma or sepsis may be accompanied by CIRCI, related to an increased inci-

From the Servei de Medicina Interna Equina and Unitat Equina, Departament de Medicina i Cirurgia Animals, Hospital Clínic Veterinari, Barcelona, Spain (Armengou, Viu, Cesarini, Jose-Cunilleras, Monreal); and the Laboratory of Biostatistics \& Epidemiology, Universitat Autònoma de Barcelona, Statistics and Methodology Support Unit, IDIBAPS, Hospital Clínic, Barcelona, Spain (Ríos). Deceased (Monreal).

This study was partially presented as oral communication at the 5th European College of Equine Internal Medicine Congress in Edinburgh (UK), February 2012.

Corresponding author: Eduard Jose-Cunilleras, DVM, PhD, DACVIM, Servei de Medicina Interna Equina, Departament de Medicina i Cirurgia Animals, Facultat de Veterinària, , Universitat Autònoma de Barcelona, 08193, Bellaterra, Barcelona, Spain; e-mail: eduard.jose.cunilleras@uab.cat.

Submitted February 22, 2012; Revised January 18, 2013; Accepted January 30, 2013.

Copyright (C) 2013 by the American College of Veterinary Internal Medicine

$10.1111 /$ jvim. 12064

$\begin{array}{ll}\text { Abbreviations: } \\ \text { ACTH } & \text { adrenocorticotropic hormone } \\ \text { BA } & \text { bile acids } \\ \text { CI } & 95 \% \text { confidence intervals } \\ \text { CIRCI } & \text { critical illness-related corticosteroid insufficiency } \\ \text { CV } & \text { coefficient of variation } \\ \text { GEE } & \text { general estimation equation } \\ \text { GGT } & \gamma \text {-glutamyltransferase } \\ \text { GLDH } & \text { glutamate dehydrogenase } \\ \text { HPA } & \text { hypothalamic-pituitary-adrenal } \\ \text { IR } & \text { insulin resistant } \\ \text { LR+ } & \text { positive likelihood ratio } \\ \text { MODS } & \text { multiple organ dysfunction syndrome } \\ \text { NEFAs } & \text { nonsterified fatty acids } \\ \text { NPV } & \text { negative predictive value } \\ \text { OR } & \text { odds ratio } \\ \text { PAS } & \text { perinatal asphyxia syndrome } \\ \text { PPV } & \text { positive predictive value } \\ \text { RAI } & \text { relative adrenal insufficiency } \\ \text { SD } & \text { standard deviation } \\ \text { SIRS } & \text { systemic inflammatory response syndrome } \\ \text { TG } & \text { triglycerides } \\ \text { UE-FHCV } & \text { Unitat Equina-Fundació Hospital Clínic Veterinari } \\ & \end{array}$

dence of shock, multiple organ dysfunction syndrome (MODS), and death in both humans ${ }^{7-9}$ and foals. ${ }^{1,3,5}$ The adrenocorticotropic hormone (ACTH)/cortisol ratio may be more indicative of HPA axis function and, therefore, a better detector of CIRCI than the absolute value of either of these hormones alone. ${ }^{2}$

Sporadic increased triglycerides (TG) concentrations have been detected in adult hospitalized sick horses without overt macroscopic lipemia. ${ }^{10}$ All of these patients had clinical and laboratory findings consistent 
with systemic inflammatory response syndrome (SIRS) and it seems, therefore, that SIRS predisposes to hypertriglyceridemia. Limited information is available with reference to normal values of plasma TG concentrations in foals. Only a reference range of plasma TG concentrations for the 1-day-old foal $(8.9-17.7 \mathrm{mg} / \mathrm{dL})$ has been published. ${ }^{11,12}$ In foals, there are only 3 reported cases of hyperlipemia unrelated to parenteral nutrition. ${ }^{13-15}$ In a recent publication, 12 healthy newborn foals had mean TG concentrations below $95 \mathrm{mg}$ / dL. ${ }^{16}$ In another study, plasma TG concentrations were assessed in a group of sick foals, with a median result of $79 \mathrm{mg} / \mathrm{dL}$ (range, $12-986 \mathrm{mg} / \mathrm{dL}$ ) for the septic group. ${ }^{6}$ Little information exists about the normal biochemistry profile of hepatic markers in equine neonates. Previous studies have reported reference ranges of several hepatic blood markers, ${ }^{12,16,17}$ but results for some other enzymes, such as glutamate dehydrogenase (GLDH), are lacking. In addition, information about biochemical markers of hepatic function in sick equine neonates is unavailable.

Several studies in sick foals have reported specific endocrine or metabolic markers, but few have tried to assess multiple endocrine and metabolic functions in a large cohort of sick equine neonates presented to a referral equine hospital.

Two main objectives have, therefore, been pursued in this study: 1) to describe metabolic and endocrine profiles in a group of septic foals and compare them with sick nonseptic and healthy foals; and, 2) to assess the relationship that metabolic and endocrine derangements may have with survival.

\section{Materials and Methods}

\section{Animals}

For this prospective study, all neonatal foals $(<21$ days old on admission) referred to the UE-FHCV of the Universitat Autònoma de Barcelona during the period from February 2005 to April 2007 were included. According to their final diagnosis, the foals were distributed into 2 main groups. A foal was included in the septic group when at least 1 of the following criteria was met: positive blood culture, septic score ${ }^{18} \geq 14$, septicemic polyarthritis, or postmortem confirmation of sepsis. The 2 nd group included all sick foals that did not meet the criteria of sepsis.

A 2 nd classification regarding outcome was made. The survivor group included all foals that left the hospital with medical consent and the nonsurvivor group included those foals that died or were euthanized because of poor prognosis. Those foals that either left the hospital or were euthanized, because of economic restraints, were not included in this classification.

Healthy neonatal foals from a stud farm nearby were included as a control group. Healthy status was assessed by clinical history, physical examination and blood test results including hematology, serum IgG concentration, and septic score $<11$.

Dams of all sick and healthy foals included in the study were examined clinically.

\section{Blood Sampling and Measured Parameters}

On admission, blood samples from sick foals were obtained either by cephalic or jugular venipuncture or from a catheter in the jugular vein, and were placed into lithium heparin plastic tubes and stored at room temperature until centrifuged within $2 \mathrm{~h}$ after blood withdrawal. When possible, a 2 nd blood sample was obtained within $24-48 \mathrm{~h}$ after admission, and a 3rd sample immediately before discharge or death. A single blood sample from each control foal was obtained. Tubes were centrifuged for $15 \mathrm{~min}$ at $1000 \times \mathrm{g}$, and plasma was removed using plastic pipettes, placed into 2 aliquots, and stored in plastic Eppendorf tubes at $-80^{\circ} \mathrm{C}$ until analyzed.

The following variables were determined for each plasma sample: TG, nonsterified fatty acids (NEFAs), glucose, creatinine, urea, c-glutamyltransferase (GGT), GLDH, insulin, cortisol, bile acids (BA), and ACTH concentrations. An automatized biochemistry analyzer ${ }^{\mathrm{a}}$ was used to determine NEFA, ${ }^{\mathrm{b}} \mathrm{BA},{ }^{\mathrm{c}} \mathrm{GLDH},{ }^{\mathrm{c}}$ $\mathrm{TG}^{\mathrm{d}}{ }^{\text {glucose }},{ }^{\mathrm{d}}$ creatinine, ${ }^{\mathrm{d}}$ urea $^{\mathrm{d}}$, and $\mathrm{GGT}^{\mathrm{d}}$ activities or concentrations. Insulin ${ }^{\mathrm{e}}$ and cortisol $^{\mathrm{f}}$ concentrations were determined using an ELISA method. ${ }^{\mathrm{g}}$ This specific insulin test has been previously validated for horses. ${ }^{19}$ ELISA assay for cortisol determination was validated following the methodology described elsewhere. ${ }^{20}$ For the calculation of intra-assay CV, a low concentration sample $(2.5 \mathrm{ng} / \mathrm{mL})$ and a high concentration sample $(70.9 \mathrm{ng} / \mathrm{mL})$ were analyzed 10 times. For the calculation of interassay $\mathrm{CV}$, the same samples were analyzed over 6 consecutive days. Mean values for intra-assay $\mathrm{CV}$ and interassay $\mathrm{CV}$ were 6.2 and $8.6 \%$, respectively. An individual sample with an initial concentration of $70.9 \mathrm{ng} / \mathrm{mL}$ was used to perform serial dilutions containing $75,50,25$, and $12.5 \%$ of the analyte to determine assay linearity. Using linear regression analysis, an $r$-value of 0.998 was obtained.

The relationship between the ELISA assay and the previously validated chemiluminescence assay ${ }^{\mathrm{h}}$ was assessed by parallel analysis of samples with different concentrations from 28 different horses, ranging from 1.3 to $97.4 \mathrm{ng} / \mathrm{mL}$. The ELISA yielded a mean $99.0 \%$ recovery of cortisol from spiked samples.

Lin's concordance coefficient and its $95 \%$ confidence interval $(\mathrm{CI})^{21}$ were used to assess agreement between both assays. This coefficient measures accuracy and precision to determine whether observed data significantly diverge from the line of perfect concordance (ie, a regression line increasing 45 degrees from the origin $[0,0]$, with a range from 0 to 1 ). Other methods, such as Passing-Bablok or Deming regression, assume theoretical conditions that are not always present. Therefore, Lin's method may be a more robust test. ${ }^{22}$ A Bland-Altman graphical representation of this analysis also was performed to detect bias (ie, differences in means between the 2 assays) or other problems that could affect concordance. ACTH was determined using a chemiluminescence technique with an automated analyzer $^{\mathrm{h}}$ that had been previously validated in horses. ${ }^{23}$ Calculations of $\mathrm{ACTH} /$ cortisol and glucose/insulin ratios also were performed.

\section{Statistical Analysis}

Data were described using median and interquartile range. Comparison of septic and nonseptic groups with control group and between nonsurvivor and survivor groups was performed using a 1-way ANOVA with a nonparametrical approach by means of rank transformation of dependent variables. On admission, and during follow-up, differences between septic and nonseptic groups were analyzed by means of a general estimating equation (GEE) methodology to account for intrasubject correlations over time, with the assumption of unstructured correlation matrix. Two models were used: one for main effects included baseline value on admission and foal age as covariate and a 2nd model included interaction of sepsis by time to evaluate differences at each point. Before GEE model analysis, missing values in any of the 3 observation times were assumed to follow a pattern, missing 
at random (MAR). Expectation-maximization (EM) algorithm considering each foal's diagnosis and prognosis was used. All values obtained were checked by an independent veterinary investigator to verify the absence of inconsistencies or errors.

An evaluation of possible cutoff values for death was performed for all of the variables assessed calculating an optimal positive likelihood ratio (LR+), defined as the ratio of sensitivity/ (1-specificity) as previously described. ${ }^{24}$ Candidates for cutoff values were those with $\mathrm{LR}+>4$. The usefulness of these candidates as independent prognostic predictors of death was evaluated by estimations of OR and their $95 \%$ confidence intervals (CI) using univariate logistic regression models. A final global approach was performed using those variables with altered values, defined as values above calculated cutoffs. In all of the analyses, a 2 -sided type I error probability of $5 \%$ was used. SPSS version 15 for Windows ${ }^{\mathrm{i}}$ was used for all statistical analyses.

\section{Results \\ Animals}

A total of 125 sick foals were included in the study. Fifty-one of 125 foals $(41 \%)$ were included in the septic group, 30 of them were females and 21 were males. Their median age was $2(0.75-5)$ days. Breed distribution was the following: Andalusian (40), crossbred (6), Arabian (4), and undetermined (1).

A total of 74 foals $(59 \%), 33$ females and 41 males, were included in the nonseptic group. Their median age was 2 (0.5-5) days and breed distribution was as follows: Andalusian (59), crossbred (7), Arabian (7), and Appaloosa (1). The nonseptic group included 43 $(58 \%)$ foals with a variety of diagnoses, such as enteritis, failure of transfer of passive immunity, acute renal failure, arthritis, intestinal obstruction, or hemolytic anemia. Thirteen foals (18\%) had a suspected but not confirmed diagnosis of sepsis or a septic score ranging between 11 and 13. During the study period, $10(14 \%)$ foals from 3 farms were diagnosed with neonatal nutritional myodegeneration and $8(11 \%)$ foals were diagnosed with perinatal asphyxia syndrome (PAS).
Regarding outcome, 121 sick foals were observed. Three foals from the septic group and 1 from the nonseptic groups were excluded because they were euthanized because of financial limitations. Therefore, 85 of $121(70 \%)$ were discharged, $36(30 \%)$ died (10 for natural reasons), and 26 were euthanized because of poor prognosis. Twenty-two survivors were classified as septic, 63 as nonseptic, and the nonsurvivors were classified as septic (26) and nonseptic (10).

In addition, 27 Andalusian-bred healthy neonatal foals from a farm in the hospital area were included as controls. There were 17 females and 10 males. Median age was 4 (2-7) days. None of them were premature.

\section{Endocrine and Metabolic Profiles on Admission (Table 1)}

Plasma TG concentration was $>100 \mathrm{mg} / \mathrm{dL}$ in $55 \%$ of the septic foals and in $34 \%$ of the nonseptic foals although no differences in the median value were observed compared with the control group. Plasma glucose concentration was lower in the septic group compared to control $(P=.002)$, although the median value was within the reference range in all groups. Median ACTH concentration of the septic group was 4.6 times higher compared to the control group $(P<.001)$. Plasma cortisol concentration was 4 times higher in the nonseptic group and 8 times higher in the septic group compared to control $(P<.001)$. Urea, creatinine, and NEFAs concentrations also were increased in nonseptic and septic foals compared to controls $(P<.001)$. GGT $(P<.05)$ and ACTH $/$ cortisol ratio $(P<.001)$ were lower in both septic and nonseptic foals compared to controls.

Regarding outcome classification, TG, creatinine, GLDH, ACTH, and cortisol $(P<.001)$, urea $(P=.04)$, and $\mathrm{ACTH} /$ cortisol ratio $(P=.02)$ had significantly higher values in the nonsurvivors than in the survivors. A possible reference interval for plasma

Table 1. Plasma concentrations and activities on admission of all variables assessed for the control, nonseptic, septic, survivor, and nonsurvivor groups.

\begin{tabular}{|c|c|c|c|c|c|}
\hline & \multirow[b]{2}{*}{ Control } & \multicolumn{2}{|c|}{ Groups According to Diagnosis } & \multicolumn{2}{|c|}{ Groups According to Outcome } \\
\hline & & Nonseptic & Septic & Survivor & Nonsurvivor \\
\hline Glucose $(\mathrm{mg} / \mathrm{dL})$ & $147.6(134.9-171.6)$ & $137.9(103.1-164)$ & $122.9(94.8-138.2)^{\mathrm{a}}$ & $134(106.5-158.6)$ & $114.2(66.6-144.4)$ \\
\hline Insulin $(\mu \mathrm{g} / \mathrm{L})$ & $0.3(0.1-0.4)$ & $0.1(0.1-0.4)$ & $0.2(0.1-0.6)$ & $0.2(0.1-0.4)$ & $0.1(0.1-0.6)$ \\
\hline Glucose/insulin ratio & $0.7(0.4-1.1)$ & $0.8(0.3-1.6)$ & $0.4(0.2-1)$ & $0.8(0.3-1.5)$ & $0.5(0.1-1.5)$ \\
\hline $\mathrm{TG}(\mathrm{mg} / \mathrm{dL})$ & $84(62-115)$ & $81(42-235)$ & $118(59-283)$ & $71(39-155)$ & $253(112-305.5)^{\mathrm{b}}$ \\
\hline NEFAs $(\mathrm{mmol} / \mathrm{L})$ & $0.2(0.2-0.3)$ & $0.6(0.4-0.9)^{\mathrm{a}}$ & $0.7(0.4-0.9)^{\mathrm{a}}$ & $0.6(0.4-0.9)$ & $0.8(0.5-0.9)$ \\
\hline Urea $(\mathrm{mg} / \mathrm{dL})$ & $15.4(13-20.9)$ & $38.2(27-47.2)^{\mathrm{a}}$ & $40.2(24.7-62.1)^{\mathrm{a}}$ & $38.1(24.2-44)$ & $52.5(28.1-84.4)^{\mathrm{b}}$ \\
\hline Creatinine $(\mathrm{mg} / \mathrm{dL})$ & $1(0.9-1.1)$ & $1.4(1-1.7)^{\mathrm{a}}$ & $1.6(1-2.6)^{\mathrm{a}}$ & $1.4(1-1.6)$ & $1.9(1.2-2.8)^{\mathrm{b}}$ \\
\hline GLDH $(\mathrm{U} / \mathrm{L})$ & $10.8(5.6-17.1)$ & $7(4.7-10.3)$ & $10.3(3.1-20.7)$ & $6.1(3.6-10)$ & $13.7(6.6-23.3)^{\mathrm{b}}$ \\
\hline GGT (U/L) & $28(23-59)$ & $23(18-35)^{\mathrm{a}}$ & $25.6(17-33)^{\mathrm{a}}$ & $24(18-29)$ & $22.5(16-39.5)$ \\
\hline $\mathrm{BA}(\mathrm{mol} / \mathrm{L})$ & $8.8(6.3-12.1)$ & $9.4(5-13.9)$ & $13.4(5.2-17.5)$ & $9.4(4.9-14.8)$ & $15.5(5.8-19.4)$ \\
\hline ACTH $(\mathrm{pg} / \mathrm{mL})$ & $18.2(15.3-31.1)$ & $22.3(13.1-32.6)$ & $83.6(22.7-158)^{\mathrm{a}}$ & $22.7(13.7-44.3)$ & $155(27.7-176.5)^{\mathrm{b}}$ \\
\hline Cortisol (ng/mL) & $8.6(2.6-14.5)$ & $36(17-52.6)^{\mathrm{a}}$ & $72.6(38.9-97.6)^{\mathrm{a}}$ & $38.9(18.3-66.1)$ & $80.7(39.2-100.7)^{b}$ \\
\hline $\mathrm{ACTH} /$ cortisol ratio & $3.1(1.7-8.6)$ & $0.9(0.4-1.5)^{\mathrm{a}}$ & $1(0.5-1.9)^{\mathrm{a}}$ & $0.8(0.4-1.4)$ & $1.5(0.5-2.7)^{\mathrm{b}}$ \\
\hline
\end{tabular}

Median (interquartile range). Statistical analysis using a nonparametric ANOVA.

${ }^{\text {a}}$ Significantly different from the control group.

bignificantly different from the survivor group. 
GLDH activity of healthy foals using the upper limit of the $95 \%$ CI was $<27.5 \mathrm{U} / \mathrm{L}$.

\section{Follow-Up Evaluation}

Estimated median values of the variables assessed during the follow-up and before either discharge or death are shown in Table 2.

An age effect in all groups was seen only for GLDH, ACTH, and glucose/insulin ratio. For some variables, there was a general difference between septic and nonseptic groups and this difference was related to sampling time. At the last sampling time, the septic group had significantly lower TG, creatinine, and insulin median concentrations $(P<.001)$ and higher glucose concentration $(P<.005)$ than did the nonseptic group, whereas ACTH/cortisol $(P=.01)$ and glucose/insulin $(P<.001)$ ratios were significantly higher in septic compared to nonseptic foals. A difference between diagnostic groups was found upon admission and at the last sampling time for ACTH $(P<.001)$. At the end of the follow-up, values in the septic group changed from significantly higher to lower compared with the nonseptic group (Table 3).

\section{Prognostic Value of Variables on Admission}

Values of LR+ for cutoff, risk of death estimations such OR, sensitivity, specificity positive predictive value (PPV), and negative predictive value (NPV) for nonsurvival for each variable on admission, as well as score, are shown in Table 4.

Cortisol was most specific, which means that almost all foals with an admission cortisol concentration $\geq 145 \mathrm{ng} / \mathrm{mL}$ were likely to die. ACTH, creatinine, and GLDH showed the highest OR for nonsurvival. A sick foal with an ACTH concentration $\geq 75 \mathrm{pg} / \mathrm{mL}$, creatinine concentration $\geq 2.3 \mathrm{mg} / \mathrm{dL}$, or GLDH activity $\geq 16.7 \mathrm{U} / \mathrm{mL}$ was more likely to die (OR, 11.8; 95\% CI, 4.6-30.2; OR, 8.9; 95\% CI, 3.2-24.6, and OR, 8.4; 95\% CI, 2.9-24.4, respectively; $P<.02$ ). In addition, a score was designed including creatinine, GLDH, and cortisol, which were the variables with $\mathrm{LR}+>4$. When a foal had 1 of these variables above the cutoff value, it had a risk 6.4 times more of dying (OR 6.4; 95\% CI, 2.37-17.34; $P<.001)$. When $\geq 2$ of these variables were altered, the risk of dying was 32.5 times more (OR, 32.5; $95 \%$ CI $6.4-165.44 ; P<.001)$ than a foal with none of the variables altered.

\section{Concordance between ELISA and Chemiluminescence Assay for Cortisol Determination}

Lin's concordance value was $0.71(95 \%$ CI, $0.52-$ $0.83)$. In the Bland-Altman graphical representation of this analysis, a consistent bias between the 2 techniques was observed, so that ELISA assay underestimates cortisol concentration compared to the chemiluminescence assay (Fig 1).

\section{Discussion}

Results obtained in this study showed that sick neonatal foals may have several simultaneous metabolic and endocrine alterations. Some of the findings, such as plasma GLDH activity and NEFAs concentration in both healthy and sick neonates and plasma GGT activity in sick foals, are described for the first time. The use of cortisol, creatinine, and GLDH in a score for survival originates from this research. At the same time, different metabolic and endocrine functions are assessed in the group of healthy and sick foals, which gives a more complete description of the metabolic and endocrine function in equine neonates.

Several enzymes and metabolites related to hepatic function and lipid metabolism were assessed in this study. GLDH is an enzyme from the hepatocyte nucleus with a short half-life in plasma. Increases in its plasma concentration are related to severe and ongoing damage in hepatocytes. ${ }^{25}$ To the authors' knowledge, this is the first report on plasma GLDH activity in a group of sick and healthy equine neonates. The reference value calculated in this population of healthy foals $(<27.5 \mathrm{U} / \mathrm{L})$ was above the reference interval of our laboratory for adult horses $(2-8 \mathrm{U} / \mathrm{L})$. Nonsurvivor foals had higher median plasma GLDH concentration than did survivors. Increased values in nonsurvivor foals may be related to acute hepatic damage related to systemic disease. In fact, several nonsurvivor foals had light microscopic hepatic lesions such as hemorrhage, inflammatory infiltration, fibrin deposits, or hepatocellular necrosis.

GGT is another liver enzyme assessed in this group of foals. Similar to previous studies, ${ }^{12,16,17}$ GGT activities obtained in healthy neonates were higher than those of adult horses. Median GGT activity of the control group was significantly higher than that of the septic and nonseptic groups. No previous information about GGT activity in sick neonatal foals is available, and the interpretation of these results therefore is difficult.

No previous data about NEFAs concentrations in healthy or sick equine neonates were found by the authors. NEFAs are generated from the hydrolysis of TG in adipocytes, which produces 1 glycerol and 3 fatty acid molecules for each TG. These fatty acids bind to albumin and generate what is known as NEFA. The increase in NEFAs concentration is probably related to the hypertriglyceridemia in sick foals and also to the increased activity of hormone-sensitive lipase caused by increased plasma cortisol concentration.

A high incidence of increased TG concentration was detected in septic foals, with a median concentration $>100 \mathrm{mg} / \mathrm{dL}$ in $55 \%$ of the cases, whereas control and nonseptic foals had a median concentration $<100 \mathrm{mg} /$ $\mathrm{dL}$. Moreover, on admission, the nonsurvivor group had a median TG concentration twice that of the survivor group. Surprisingly, foals in the septic group ultimately had a TG concentrations within the normal reference range, and even lower than that observed in 


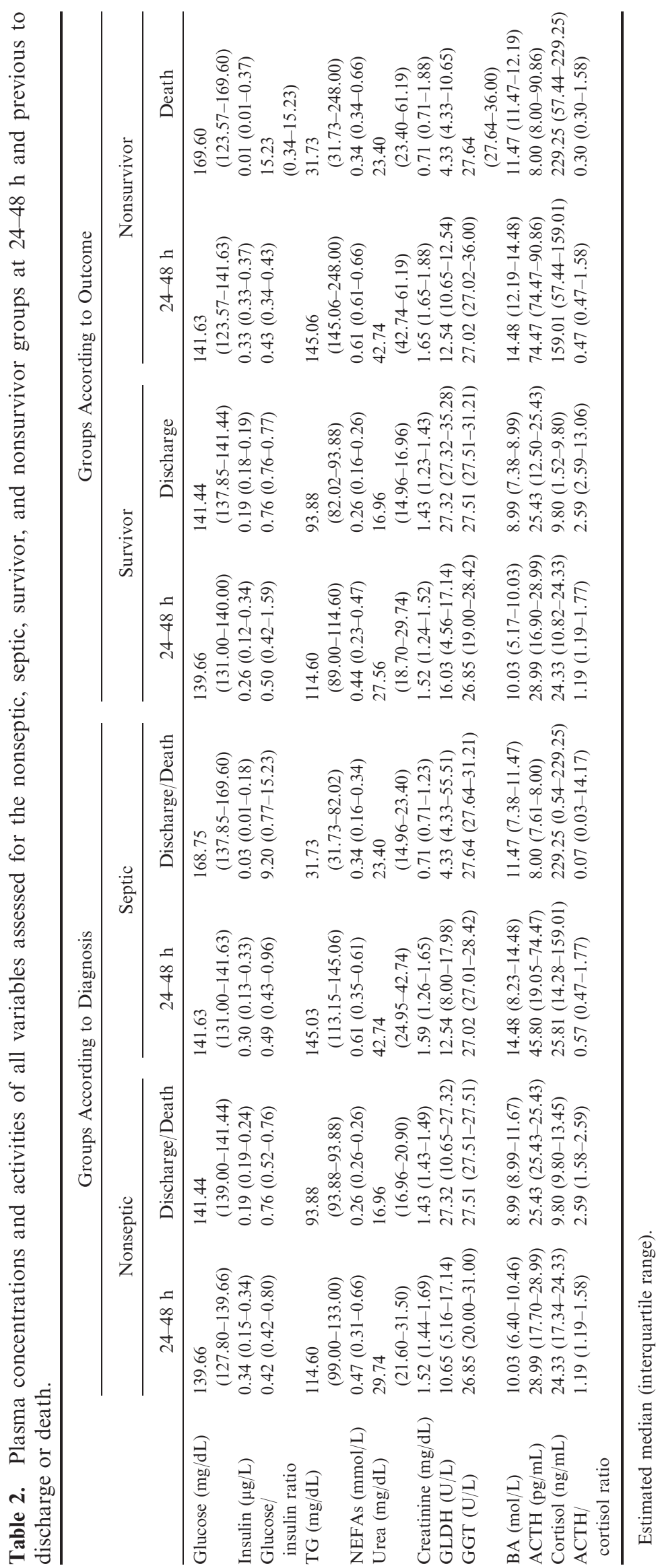


the nonseptic group. In a recent study, both septic and nonseptic foals had TG concentrations significantly higher than did healthy foals. ${ }^{6}$ Considering the outcome, overall nonsurvival was more likely, with plasma TG concentrations $<60 \mathrm{mg} / \mathrm{dL} .{ }^{6}$

In adult horses, TG concentrations $>100 \mathrm{mg} / \mathrm{dL}$ may be related to alterations in the fatty acids metabolism. ${ }^{10}$ In the foals studied here, an increase in plasma TG concentration may be secondary to a decrease in energy intake. Interestingly, macroscopic lipemia was not seen in the samples from those 5 foals with TG concentrations $>500 \mathrm{mg} / \mathrm{dL}$. Moreover, none of the mares had increased plasma TG concentrations or signs of clinical disease (data not shown). These findings are similar to those reported in a previous study, in which hypertriglyceridemia was detected in some sick adult horses without apparent serum opacity. ${ }^{10}$

Regarding glucose regulation, no clinically relevant alterations were observed in median glucose concentrations. Alterations in plasma glucose concentration have been observed in patients with sepsis. Hypoglycemia has been related to septicemia, nonsurvival, and inability to nurse neonatal foals. ${ }^{4,6}$ Although septic foals had a median glucose concentration significantly lower than control group, this concentration was within the normal reference range. The lack of alterations of glucose concentration could be because of early presentation to hospital, medications, and feeding administered before referral, decreased stress response, or both.

Insulin concentrations showed no differences among groups on admission, although a significant decrease during hospitalization was seen in the septic group compared to the nonseptic group. Increased insulin concentrations in septic foals have been related to the risk of dying. ${ }^{6}$ Glucose/insulin ratio was calculated in an attempt to detect insulin resistance (IR). During follow-up, and compared to the nonseptic foals, this ratio was increased in septic foals, which is consistent with an increase in insulin sensitivity of the former over time. Insulin resistance in foals only has been assessed in healthy unweaned foals. ${ }^{26}$ In another recent study, insulin concentrations were significantly lower in sick and septic foals compared with healthy foals. ${ }^{6}$ There are several reports describing IR and hyperglycemia both in humans and adult horses suffering from sepsis and endotoxemia. ${ }^{27,28}$ Considering the current information available, insulin appears to have a normal physiologic response in sick foals, and it would therefore be incorrect to extrapolate the observations about insulin derangements in populations of adult horses and human patients to foals. More research on insulin regulation and sensitivity in sick foals is necessary to interpret these results.

Some published studies state that the cortisol concentrations in septic foals were increased, compared to those in control foals, probably as a consequence of an endocrine response to the disease. ${ }^{1-3}$ This increase may be because of increased catecholamines in a stressful situation and SIRS related to septicemia. Median cortisol concentration in the control group was clearly lower than the reported normal values. 
Table 4. LR+, sensitivity, specificity, PPV, NPV, OR, and $P$-value of those variables useful as independent prognostic predictors of death.

\begin{tabular}{|c|c|c|c|c|c|c|c|}
\hline Parameter & $\mathrm{LR}+$ & Sensitivity & Specificity & PPV & NPV & OR $(95 \% \mathrm{CI})$ & $P$-Value \\
\hline Creatinine $\geq 2.3 \mathrm{mg} / \mathrm{dL}$ & 5.4 & 44.4 & 91.8 & 69.6 & 79.6 & $\begin{array}{l}1 \\
8.9(3.23-24.6)\end{array}$ & $<.001$ \\
\hline $\mathrm{GLDH} \geq 16.7 \mathrm{U} / \mathrm{L}$ & 5.5 & 38.9 & 92.9 & 70.0 & 78.2 & $8.4(2.88-24.35)$ & $<.001$ \\
\hline Cortisol $\geq 145 \mathrm{ng} / \mathrm{mL}$ & 4.7 & 16.7 & 96.5 & 66.7 & 73.2 & $\begin{array}{l}1 \\
5.5(1.29-23.25)\end{array}$ & .0215 \\
\hline $\mathrm{ACTH} \geq 75 \mathrm{pg} / \mathrm{mL}$ & 5.2 & 61.1 & 88.2 & 68.8 & 84.3 & $\begin{array}{c}1 \\
11.8(4.6-30.18)\end{array}$ & $<.001$ \\
\hline ACTH/Cortisol $\geq 1.9$ & 4.4 & 36.1 & 91.8 & 65.0 & 77.2 & $\begin{array}{l}1 \\
6.3(2.25-17.64)\end{array}$ & $<.001$ \\
\hline \multicolumn{8}{|c|}{ Number of parameters above cutoff value ${ }^{a}$} \\
\hline $\begin{array}{l}0 \\
1\end{array}$ & 4.0 & 66.7 & 83.5 & 63.2 & 85.5 & 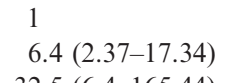 & $\begin{array}{l}<.001 \\
<.001\end{array}$ \\
\hline 2 or 3 & & & & & & $32.5(6.4-165.44)$ & $<.001$ \\
\hline
\end{tabular}

${ }^{\mathrm{a}}$ This score included only creatinine, GLDH, and cortisol.

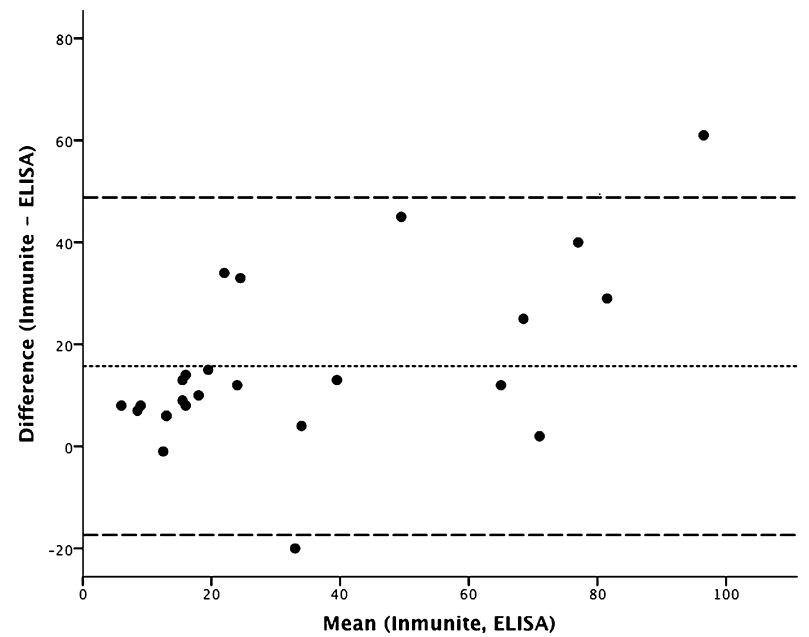

Fig 1. Bland-Altman graphical representation of the comparison between ELISA and chemiluminescence assays for cortisol determination. Horizontal dashed lines represent upper and lower limits of agreement.

However, on admission, septic and nonseptic groups had median cortisol concentrations 8 and 4 times higher, respectively, than the control group. Regarding the nonsurvivor group, the median cortisol concentration was almost 10 times that of the control group. Therefore, a clear relationship was seen among cortisol, illness, and survival. Cortisol is one of the most difficult variables to interpret because measurements will be affected by time of day, short-term fluctuations, and how accustomed the horse is to its environment. ${ }^{29}$ In neonatal foals, it also depends on hours since birth, adrenal gland maturity, and illness status. ${ }^{1}$ In this study, an ELISA technique was used for cortisol determination. When compared with a chemiluminescence assay ${ }^{\mathrm{h}}$ previously validated in horses, a bias was observed. The ELISA assay consistently underestimates cortisol concentration by $15.7 \mathrm{ng} / \mathrm{mL}$ compared with the chemiluminescence assay. Undoubtedly, it would be incorrect to compare results among different populations or techniques without taking all of these variables into account.

Median plasma ACTH concentrations of the septic and nonsurvivor groups were more than 4 times higher than the control group, and no differences were seen between the nonseptic and control groups. In previous studies, plasma concentrations of ACTH in septic foals were significantly higher than those of healthy foals. ${ }^{1-3,5}$

CIRCI is defined as an inadequate cellular corticosteroid activity for the severity of the patient's illness. ${ }^{7}$ Although this dysfunction is difficult to assess in the absence of an exogenous corticotrophin or ACTH stimulation test, the $\mathrm{ACTH} /$ cortisol ratio has been considered a useful indicator of the function of the HPA axis as compared to the absolute concentration of each hormone. ${ }^{2}$ Median $\mathrm{ACTH} /$ cortisol ratios in the septic and nonseptic groups were significantly lower than in the control group, which indicates that adrenal gland response to ACTH stimulation is not decreased in this population of sick foals on admission. However, foals in the nonsurvivor group had median $\mathrm{ACTH} /$ cortisol ratios higher than those of survivors. These results differ somewhat from those obtained in previous studies, in which septic foals had higher $\mathrm{ACTH} /$ cortisol ratios than did healthy foals, indicating the presence of CIRCI at the level of the adrenal gland. Moreover, on admission, as reported here, ACTH/cortisol ratio was higher in septic nonsurviving foals than in survivors, demonstrating prognostic value. ${ }^{1-3}$ During follow-up, this ratio increased in the septic group compared to nonseptic group because of an important increase in cortisol concentration and a moderate decrease in ACTH concentration compared to admission results. Therefore, although the ACTH/ cortisol ratio is significantly increased after admission, in the authors' opinion this cannot be interpreted as HPA axis dysfunction.

In a recent study, in which the HPA axis was assessed in healthy foals, it was concluded that foal age is important in HPA axis assessment because corti- 
sol, ACTH, and $\mathrm{ACTH} /$ cortisol ratio were higher at birth. ${ }^{5}$ In a former study, cortisol concentrations in healthy foals did not vary with age. ${ }^{1}$ In the statistical model employed in this study, an age effect for estimations of ACTH concentrations was observed which may have partially affected some of the results obtained. An age effect for the estimations of cortisol concentration and $\mathrm{ACTH} /$ cortisol ratio was not observed.

A major limitation of this study is the effect that the methodology employed may have had on ACTH stability and, consequently, on ACTH concentration and calculated ACTH/cortisol ratio. Sample collection into lithium heparin tubes, time of the sample at room temperature, storage, and analytic technique may have influenced the relatively low ACTH concentrations observed in foals when carrying out this study. ACTH determination is better performed using plasma obtained in plastic tubes containing EDTA, instead of lithium heparin, and the time at room temperature of the sample should be minimal. Because of this limitation, the $\mathrm{ACTH} /$ cortisol ratio was not included in the prognostic score, although it had a higher OR for likelihood of nonsurvival than cortisol itself. Despite these methodology limitations, the procedure was identical for all the groups included. Therefore, if these limitations influenced the ACTH concentrations, the effect would have been the same in all groups. Differences in ACTH results, observed in this and other studies of adrenal function in sick foals, also may be related to these methodology problems. ACTH may have undergone degradation, and results obtained therefore may be mistakenly low. This possibility limits the comparison of the results obtained from those of other studies. However, the tendencies or comparisons among groups are similar to those of previous studies. Information could therefore be extrapolated and shared regarding the effect that disease and outcome may have on ACTH concentrations rather than focusing on absolute results (ie, whether the ACTH concentration increases or decreases related to septicemia).

Finally, blood markers of renal function are commonly analyzed in sick foals. As expected, in this study, azotemia was present in both groups of sick foals on admission. Azotemia may be caused by prerenal or renal factors, because most foals with hypovolemia, hypotension, infection, secondary muscular lesion, PAS, or immaturity. ${ }^{30}$ Other possible causes in neonatal foals are maternal problems such as placental dysfunction. ${ }^{31}$ The relationship of increased creatinine concentration with nonsurvival may be because of tissue hypoperfusion and renal damage. Previous literature differs with regard to the relationship between creatinine concentration and survival. In several reports, the creatinine concentration of hospitalized neonatal foals was related to survival, but it was included in the final model to estimate the probability of discharge in only two of them. ${ }^{32,33}$

In conclusion, endocrine and metabolic derangements are present in sick foals, although some of the results obtained are different from those expected con- sidering previous information in septic veterinary and human patients. Septic neonatal foals tend to had hypertriglyceridemia. Although it does not seem to cause systemic derangements, such as fatty infiltration, it may be related to poor prognosis. Creatinine, GLDH, and cortisol are variables that could be included in the routine analytical panel of sick newborn foals because of their association with outcome. Caution should be exercised in the interpretation of the score obtained and not applied to the general population of newborn foals.

\section{Footnotes}

a Olympus AU400, Olympus Corporation, Ireland

b NEFA C, Wako Chemicals GmbH, Neuss, Germany

${ }^{\mathrm{c}}$ Randox, Ardmore, UK

d Olympus System Reagent, Olympus, Ireland

e Mercodia Equine Insulin ELISA, Mercodia AB, Uppsala, Sweden

${ }^{\mathrm{f}}$ Cortisol ELISA, DRG Instruments, Marburg, Germany

${ }^{g}$ ELISA Labsystems iEMS Reader/Dispenser MF, Labsystems Oy, Helsinki, Finland

${ }^{\mathrm{h}}$ Immulite, Siemens, Los Angeles, CA

${ }^{\mathrm{i}}$ SPSS Inc, Chicago, IL

\section{Acknowledgment}

Conflict of Interest Declaration: Authors disclose no conflict of interest.

\section{References}

1. Gold JR, Divers TJ, Barton MH, et al. Plasma adrenocorticotropin/cortisol, and adrenocorticotropin cortisol ratios in septic and normal-term foals. J Vet Intern Med 2007;21:791-796.

2. Castagnetti C, Rametta M, Tudor Popei R, et al. Plasma levels of ACTH and cortisol in normal and critically-ill neonatal foals. Vet Res Commun 2008;32(Suppl 1):S127-S129.

3. Hurcombe SDA, Toribio RE, Slovis N, et al. Blood arginine vasopressin, adrenocorticotropin hormone, and cortisol concentrations at admission in septic and critically ill foals and their association with survival. J Vet Intern Med 2008;22:639-647.

4. Hollis AR, Furr MO, Magdesian KG, et al. Blood glucose concentrations in critically ill neonatal foals. J Vet Intern Med 2008;22:1223-1227.

5. Hart K, Slovis N, Barton M. Hypothalamic-pituitaryadrenal axis dysfunction in hospitalized neonatal foals. J Vet Intern Med 2009;23:901-912.

6. Barsnick RJIM, Hurcombe SDA, Smith PA, et al. Insulin, glucagon, and leptin in critically ill foals. J Vet Intern Med 2011;25:123-131.

7. Marik PE, Pastores SM, Annane D, et al. Recommendations for the diagnosis and management of corticosteroid insufficiency in critically ill adult patients: Consensus statements from an international task force by the American College of Critical Care Medicine. Crit Care Med 2008;36:1937-1949.

8. Annane D, Sebille V, Troche G, et al. A 3 level prognostic classification in septic shock based on cortisol levels and cortisol response to corticotropin. J Am Med Assoc 2000;283:1038-1045. 
9. Marik P. Critical illness related corticosteroid insufficiency. Chest 2009;135:181-193.

10. Dunkel B, McKenzie HC III. Severe hypertriglyceridaemia in clinically ill horses: Diagnosis, treatment and outcome. Equine Vet J 2003;35:590-595.

11. Knottenbelt DC. Perinatal review. In: Knottenbelt DC, ed. Equine Neonatology: Medicine and Surgery. London: WB Saunders; 2004:1-18.

12. Bauer JE, Asquith RL, Kivipelto J. Serum biochemical indicators of liver function in neonatal foals. Am J Vet Res 1989;50:2037-2040.

13. Gilbert RO. Congenital hyperlipaemia in a Shetland pony foal. Equine Vet J 1986;18:498-500.

14. Hughes KJ, Hodgson DR, Dart AJ. Hyperlipaemia in a 7-week-old Miniature pony foal. Aust Vet J 2002;80:350351.

15. Tan RH, Hughes KJ, Hodgson DR. Hyperlipaemia, neonatal isoerythrolysis and hepatocellular necrosis in a 3-day-old Thoroughbred foal. Aust Vet J 2005;83:740-741.

16. Barton MH, LeRoy BE. Serum bile acids concentrations in healthy and clinically ill neonatal foals. J Vet Intern Med 2007;21:508-13.

17. Gossett KA, French DD. Effect of age on liver enzyme activities in serum of healthy Quarter Horses. Am J Vet Res 1984;45:354-356.

18. Brewer BD, Koterba AM. Development of a scoring system for the early diagnosis of equine neonatal sepsis. Equine Vet J 1988;20:18-22.

19. Öberg J, Bröjer J, Wattle O, et al. Evaluation of an equine-optimized enzyme-linked immunosorbent assay for serum insulin measurement and stability study of equine serum insulin. Comp Clin Pathol 2012;21:1291-1300.

20. Boutet P, Heath F, Archer J, Villiers E. Comparison of quantitative immunoturbidimetric and semiquantitative latexagglutination assays for D-dimer measurement in canine plasma. Vet Clin Pathol 2009;38:78-82.

21. Lin LI. A concordance correlation coefficient to evaluate reproducibility. Biometrics 1989;45:255-268.
22. McBride GB. A proposal for strength-of-agreement criteria for Lin's Concordance Correlation Coefficient. NIWA Client Report 2005; HAM2005-062.

23. Perkins GA, Lamb S, Erb HN, et al. Plasma adrenocorticotropin $(\mathrm{ACTH})$ concentrations and clinical response in horses treated for equine Cushing's disease with cyproheptadine or pergolide. Equine Vet J 2002;34:679-85.

24. Deeks JJ, Altman DG. Diagnostic tests 4: Likelihood ratios. BMJ 2004;329:168-169.

25. Stockham SL. Enzymes. In: Stockham SL, Scott MA, eds. Fundamentals of Veterinary Clinical Pathology, 2nd ed. Iowa: Blackwell Publishing; 2008:654-655.

26. George LA, Staniar WB, Treiber KH, et al. Insulin sensitivity and glucose dynamics during pre-weaning foal development and in response to maternal diet composition. Domest Anim Endocrinol 2009;37:23-29.

27. Van Cromphaut SJ, Vanhorebeek I, Van den Berghe G. Glucose metabolism and insulin resistance in sepsis. Curr Pharm Des 2008;14:1887-1899.

28. Toth F, Frank N, Elliott SB, et al. Effects of an intravenous endotoxin challenge on glucose and insulin dynamics in horses. Am J Vet Res 2008;69:82-88.

29. Irvine $\mathrm{CHG}$, Alexander SL. Factors affecting the circadian rythm in plasma cortisol concentrations in the horse. Domest Anim Endocrinol 1994;11:227-238.

30. Chaney KP, Holcombe SJ, Schott HC 2nd, et al. Spurious hypercreatininemia: 28 neonatal foals (2000-2008). J Vet Emerg Crit Care 2010;20:244-249.

31. Brewer BD. The urogenital system: Renal disease. In: Koterba AM, Drummond WH, Kosch PC, eds. Equine Clinical Neonatology. Philadelphia: Lea \& Febiger; 1990:446-458.

32. Bedenice D, Heuwieser W, Solano M, et al. Risk factors and prognostic variables for survival of foals with radiographic evidence of pulmonary disease. J Vet Intern Med 2003;17:868-875.

33. Rohrbach BW, Buchanan BR, Drake JM, et al. Use of a multivariable model to estimate the probability of discharge in hospitalized foals that are 7 days of age or less. J Am Vet Med Assoc 2006;228:1748-1756. 